\title{
Dust Properties of Planetary Nebulae
}

\author{
Stefan Kimeswenger \\ Institut für Astrophysik, Leopold-Franzens Universität Innsbruck, \\ Technikerstraße 25, A-6020 Innsbruck, AUSTRIA
}

\begin{abstract}
Shells of planetary nebulae (PNe) are usually modeled applying photoionization only although there is a significant fraction of dust embedded in the nebular shells. The calculated temperature and dustto-gas ratio from IRAS colors is "faked" by the IR flux continuum contribution from the gas and by transiently heated dust grains. Also the effects of grain growth time and thus maximum grain size lead to strong deviations from the model input values, adopting the IRAS data fits.
\end{abstract}

I present here a sample of synthetic models of PNe along evolutionary tracks. The shells assumed are not result of sophisticated hydrodynamic models, but taken from assumptions of homogeneous spherical expanding shells with a nonlinear decrease of the filling factor with age. A $0.524 \mathrm{M}_{\odot}$ and a $0.625 \mathrm{M}_{\odot}$ evolutionary track by Blöcker (1995) were used to derive the central star luminosity, and the age. The expansion velocities, $20 \mathrm{~km} / \mathrm{s}$ and $80 \mathrm{~km} / \mathrm{s}$ for low mass stars and for massive ones, respectively, were adopted from the average values found in Weinberger (1989) for nebulae with matching central stars. Also the decrease of the filling factors as function of time were chosen to fit to the average measurements of the [SII] densities vs. the $6 \mathrm{~cm}$ radio flux from various literature sources. The dust codes used here include the UV extinction as well as the NIR and MIR emission due to fluctuating temperature in small particles (Koller \& Kimeswenger 2001). For the description of the gas component, CLOUDY was used as a "partner subroutine".

I obtained the IR emission as function of the maximum grain size, geometry (respectively age) and central star luminosity at constantly retained dust-to-gas ratio. Krüger et al. (1996) and Patzer et al. (1998) showed that the maximum grain size depends strongly on the wind speed and the chemical abundance of the CNO elements during the short period of grain growth. Simis et al. (2001) show that even at the same model a modulation between two cycles of grain production may occur. Thus the "phase", of those cycles when expelling the shell is also of importance. Thus we have to assume a wide range of maximum grain sizes in the dust. The "classical" method (Potasch et al. 1984; Stasinska \& Sczcerba 1999) to derive the dust temperature and the gas-to-dust ratio was applied to the spectral energy distribution (SED) models. At the early and at the very late stages of the evolution also the effects of using transiently heated dust grains were significant.

The calculated temperature and dust-to-gas ratio is "faked" by the IR flux continuum contribution from the gas, by the transiently heated dust, and by the duration of the grain growth phase (respectively by changes in $a_{\mathrm{MAX}}$ ). Applying 


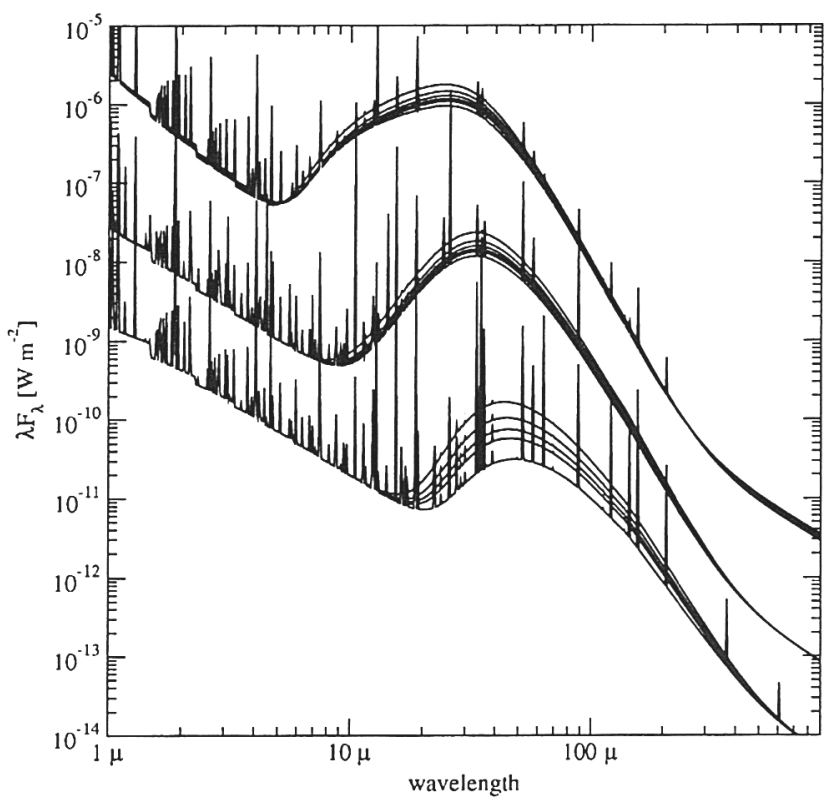

Figure 1. The sequence of models (left panel) of the $0.625 \mathrm{M}_{\odot}$ central star - from top to bottom at 1000 years, 35000 years and 80000 after leaving the AGB. The subsets are defined by the maximum grain size $a_{\text {MAX }}$ of $0.1,0.2,0.3,0.4,0.5$ and $0.75 \mu \mathrm{m}$. The changes of the SED are clearly visible. Thus the "temperature" derived from the 25 and $60 \mu \mathrm{m}$ flus and the gas-to-dust ratio from it does not give the real values.

this model grid to the nebula NGC 2438 (using the density distribution derived for the optical images/spectra; Armsdorfer, Kimeswenger \& Rauch, these proceedings) leads to $a_{\mathrm{MAX}}=0.3 \mu \mathrm{m}, \mathrm{L}_{\mathrm{CSPN}}=430 \mathrm{~L}_{\odot}$ and a dust-to-gas ratio of 180.

These investigations show that there is a need for a reappraisal of the $\mathrm{MG} / \mathrm{MD}$ ratio, and via the UV extinction thus for the spectroscopic diagnostic diagrams.

\section{References}

Blöcker, T. 1995, A\&A, 297, 727

Pottasch, S.R., Baud, B., Beintema, D., et al. 1984, A\&A, 138, 10

Stasinska, G., \& Szczerba, R. 1999, A\&A, 352, 297

Koller, J., \& Kimeswenger, S. 2001, ApJ, 559, 415

Krüger, D., Patzer, A.B.C., \& Sedlmayr, E. 1996, A\&A, 313, 891

Patzer, A.B.C., Gauger, A., \& Sedlmayr, E. 1998, A\&A, 337, 847

Simis, Y.J.W., Icke, V., \& Dominik, C. 2001, A\&A, 371, 205

Weinberger, R. 1989, A\&AS, 78, 301 